\title{
Care programme approach: relapsing or recovering?
}

\author{
REVISITING... MAKING CARE PROGRAMMING WORK \\ David Kingdon \& Shabbir Amanullah
}

Abstract The care programme approach (CPA) has become an accepted part of clinical practice, despite the
continuing lack of strong direct evidence of its value. Guidance from the Department of Health has
refined the original requirements, which were to ensure health and social care assessment, discharge
from hospital to appropriate accommodation with necessary support, appointment of a mental health
professional to draw up a care plan, and coordination of its implementation with necessary follow-up.
The CPA now specifies that care plans include provision, as necessary, for risk assessment and
management, employment, leisure, accommodation and plans to meet carers' needs. Levels of care
have been simplified to 'standard' and 'enhanced'. In future it will need to incorporate issues arising
from the development of specialist teams as part of the National Health Service Plan, concern about the
physical healthcare of those subject to it and the continuing development of psychosocial interventions.

Kingdon \& Amanullah revisit an article published over a decade ago in APT (Kingdon, 1994a). The 1994 article is available on our website (http://apt.rcpsych.org), as a data supplement to the online version of the present submission.

The care programme approach (CPA) has not been the most popular policy emanating from the Department of Health over the past couple of decades, during which it has shown an increasing interest in mental health. But it can be argued that it is as important as any and certainly has had a major influence on staff, patients and carers. It was always intended to describe and put into place good clinical practice as community services increasingly replaced hospital-centred ones. This meant that services prioritised the provision of services in relation to need, and ensured that discharge from hospital was to appropriate accommodation, with follow-up provided as needed. Versions of the CPA have now been adopted in Wales, Scotland and Ireland as they have recognised the need for a defined multidisciplinary clinical policy to organise care in community settings.

The basic requirements of the CPA have not changed since the Department of Health first introduced it in 1991 (Department of Health, 1990). It still is intended to provide a safety net of care for people with mental illness accepted by mental health services, by ensuring that each person has a care plan which is reviewed regularly or as necessary and a mental health worker who coordinates care delivery. The implementation of the CPA has changed, as have some of the terms. We now refer to keyworkers as care coordinators. Keyworker was a more generic term than envisaged within the CPA, and used in specific mental health units such as day hospitals and wards; it also led to confusion with 'key workers' from other agencies, for example social services and housing associations. New guidance has appeared which has attempted to clarify certain areas and emphasise specific concerns (Department of Health, 1999):

- risk assessment and management

- employment

- leisure

- accommodation

- plans to meet carers' needs.

Levels of CPA have been simplified to:

- enhanced - in practice, for those whose care needs are best served by regular multidisciplinary review meetings

- standard - where such meetings are unnecessary.

David Kingdon is Professor of Mental Health Care Delivery at the University of Southampton (Royal South Hants Hospital, Southampton SO14 0YG, UK. E-mail: dgk@soton.ac.uk) and an honorary consultant with Hampshire Partnership Trust. His research interests include psychosocial and healthcare interventions in severe mental illness. Shabbir Amanullah is a specialist registrar on the Wessex Higher Training Scheme. His interests are qualitative methods of research and medical education. 


\section{Who should we treat? \\ Defining severe mental illness}

A specific definition of severe mental illness has never been given because of the complexity of specifying a point on the continuum of 'illness' that is relevant to individual patients, who have widely varying needs, and to services that have varying levels of resources. However, in Building Bridges (Department of Health, 1995) a framework for developing such criteria and making decisions about resource allocation was proposed. This involves consideration of:

- safety

- need for informal or formal care

- diagnosis

- disability

- duration of illness.

Diagnosis does feature but has marginal, if any, meaningful contribution to make. Where diagnosis has been used, psychotic disorders have tended to take prominence. Given that many people with psychoses at some stage in their lives can make excellent recoveries, this is too broad; conversely many people with other disorders, for example anorexia, depression and obsessive-compulsive disorder, may be more severely mentally ill. There is now a programme of work designed to operationalise this framework in the form of criteria to use in determining eligibility for services (Institute of Psychiatry, 2005).

\section{Prioritising referrals}

Because of the limited financial and personnel resources available, mental health services have had to develop criteria to prioritise who is accepted for assessment and subsequent allocation to community mental health team members. A single point of entry to include those referred to psychiatrists has been advocated. For each referral, the team would consider the appropriateness of offering a service and decide whether a medical assessment or assessment by another team member is needed. This can make best use of scarce medical time and allow the psychiatrist to become more a 'consultant' to the team, as is being proposed (Department of Health, 2004).

\section{Has anything changed?}

Progress has occurred since the CPA was introduced. In a review of health and social services, the Social Services Inspectorate (1999) found, in contrast to earlier inspections:
- a good understanding of the use of the CPA for assessment and care planning;

- joint health authority and social services department strategies in place or in development;

- services developing in a more flexible way;

- extensive involvement of users and carers in care planning.

The authorities that had made most progress in implementing the Department of Health's (1995) guidance on inter-agency care of people with severe mental illness had:

- pooled or shared budgets (within current legal boundaries)

- shared management

- user-focused systems

- a committed, strategic lead, with all professionals involved.

Interviews with service users found that most receiving CPA were seeing a psychiatrist regularly but were also continuing to visit their GP about mental health needs more frequently than they attended psychiatric out-patient appointments. This suggests that at least some duplication of service between primary and secondary healthcare may be occurring.

\section{Implementation}

\section{The integrity of the policy}

Inevitably there is disparity nationally in implementation of the CPA. This has led some to describe it as a failed policy (Simpson et al, 2003), but the claim seems premature in the absence of any equally wide-ranging alternative strategy to ensure that people with severe mental illnesses receive the care they require. There has also been increasing professional acceptance of what was always intended to be a clinical intervention. The Department of Health first issued guidance on community care of severely mentally ill people after the professional bodies could not agree on a joint response to the Spokes inquiry (Department of Health and Social Security, 1988). The Spokes inquiry was therefore the direct stimulus for the development of the CPA. The one credible alternative considered at the time was that teams rather than individual care coordinators take responsibility for patients. This is the model used by the Program for Assertive Community Treatment in Wisconsin (Allness \& Knoedler, 1998), from which some of the principles of the CPA were derived. However, the group of patients covered by the CPA is much broader than that covered by PACT teams and the potential for blurring of responsibilities is greater. 
Whatever the approach, however, it seems important that a mental health team should be available to support individuals, for example by providing a forum for discussion of difficult clinical cases, assistance for individuals required to make difficult decisions and cover when individual members are unavailable.

\section{Problems of interpretation}

Implementation has certainly been patchy, but pressure from the Department of Health Inspectorates and Mental Health Act Commission on one hand and from users and carers who want care plans and clear points of contact on the other has had some effect. However, the use of the CPA as justification for local management initiatives has often caused it to fall into disrepute. For example, the introduction of lengthy, complex and poorly validated assessment instruments for generic use, and specifically for risk management, has overwhelmed practitioners in underresourced services that often have vacant posts. Although these have been introduced as requirements of CPA they are not in fact compulsory for all patients. Useful initiatives such as advanced directives (Henderson et al, 2004) have been submerged under a mass of associated initiatives interpreted as compulsory for all, whereas they are intended for selective implementation. Similarly, multidisciplinary reviews can be valuable in coordinating care but they must be conducted selectively because of their cost in time of all the individuals involved. In many circumstances individual discussions between team members and psychiatrists may be more efficient. Involvement of users and carers is important, as their views and needs should be taken fully into account in decision-making. But this can be done appropriately, often by individual discussion before or after the psychiatrist and other team member have met.

The experience of one region in implementation of the CPA is outlines in Box 1.

\section{Supervision registers and supervised discharge}

Government policy initiatives such as supervision registers and supervised discharge may have had an impact on the CPA. As both target community patients most at risk of harm to self or others, identification of this group may have propelled services into more systematic assessment of the risk and needs of individuals in their care. It may also have accelerated the process by which people are identified as requiring enhanced CPA and regular multidisciplinary review is instigated, but mental health staff have never seen supervision registers in

\section{Box 1 The problems of implementation: Southampton's experience}

A simple CPA system involving minimal paperwork was introduced in Southampton's mental health services in 1996. However, new guidance on care coordination (Department of Health, 1999) and a merger of the mental health services into the Hampshire Partnership Trust initiated a root and branch review of CPA implementation. This focused on paperwork and resulted in widespread consultation, wholesale disruption and potentially overwhelming bureaucratisation. The CPA documentation expanded from two sides of A4 to a pack more than an inch thick. Messages from above were mixed: another trust revealed a similar overweight stack of paperwork, derided and erratically used by staff but given 5-star rating by the Commission for Health Improvement.

The review was used to bring in structured assessments, advance directives, cumbersome risk assessment tools and a range of administrative documents for clinical staff to complete. Fortunately, the objectives established by the new Department of Health (1999) guidance are more specific, concerns expressed by patients and carers have been identified and ways to meet both have been found without overwhelming patients and care coordinators.

In the current system, assessments in different services vary: rehabilitation, child and adolescent, old age, adult and forensic services have differing processes and continue to use these flexibly. There are common elements, and advance directive forms and assessment tools, for example, are available if staff wish to use them. Risk assessments need to be documented and a 'risk prompt sheet' has been developed to support this process. 'Passport details' (personal data) are collected separately but the CPA review form is now back to two sides of A4 (copies of documentation are available from author) and is used in enhanced CPA for multidisciplinary reviews. Some staff also use it in standard CPA, but most simply write a letter containing relevant details, copied to the patient. Nobody seems to complain about CPA any more... for the time being. 
themselves as having much value (Bindman et al, 2000). In trusts in which the CPA has been demonstrated to be fully implemented supervision registers are no longer a requirement.

Supervised discharges have been viewed as more useful but only by the small proportion of psychiatrists $(18 \%)$ who have used them (Franklin et al, 2000). The provisions for community treatment in the proposed new Mental Health Act will replace supervised discharges.

\section{New developments}

Then along came the NHS Plan, with its proposals for early intervention services, assertive outreach and crisis resolution/home treatment teams (Department of Health, 2002). The development of these teams may be affecting criteria used but not the fundamental principles of the CPA. Early intervention services should be reaching people at an early stage in their psychotic illness - and therefore they should not have met criteria for 'severe mental illness. Implementation of the CPA while patients are with these teams, which generally have lower case-loads, is clearly their responsibility and relatively straightforward. It becomes particularly important, however, when patient transfer or coworking with other teams (e.g. community mental health teams and substance misuse teams) occur. The CPA provides a structure to ensure that responsibility is defined, i.e. which individual is care coordinator, and planning is comprehensive, with the user and carers at the centre of the process. There is a danger that the mushrooming of teams will lead to increasing gaps in service and disputes about responsibility for patients, exacerbating difficulties at existing interfaces between child, adult and old age services and learning disability, forensic and substance misuse teams. The CPA provides a process to ensure that threse problems do not occur - when it is effectively implemented.

The incorporation of psychosocial interventions such as family work, cognitive therapy and vocational supports is fully compatible with the CPA. Indeed, the CPA can ensure that therapists working with people with severe mental illness are acting as part of a team, rather than detached from other mental healthcare support. 'Semi-detached' psychologists and nurse therapists working in isolation can end up working in opposite directions to team members. For example, as patients talk more about symptoms to therapists, they may also do so to their psychiatrist. If the psychiatrist is not aware that revelation of these symptoms is due to improved communication resulting from psychotherapy, he or she might alter medication dosage or regimens to deal with them, with potential negative effects on, for example, motivation and communication. Direct support from psychiatrists and care coordinators in CPA reviews in negotiating and supporting patients with homework assignments and reinforcing the value of family or individual work can also be synergistic. Collaborative discussions and explanations about medication use can be particularly important to adherence to treatment regimens.

Physical healthcare of people with serious mental illness is belatedly receiving greater attention with concerns about the adverse effects of medication and the intrinsic effects of mental health problems (e.g. through amotivation), and addressing these needs through the CPA process is an increasing priority.

\section{Conclusions}

Government intrusions into clinical practice are inevitably unpopular and resisted by clinical staff. However, the CPA is accepted by the professional organisations, including the Royal College of Psychiatrists (2004), as good practice. Understanding what it is (Kingdon, 1994a,b, 1998) and what it is not is important to its implementation. Clinical staff need to understand the CPA to ensure that it is not being used as a Trojan Horse for illthought through management initiatives and bureaucratisation but that it is being used efficiently to ensure that patients who most need services receive them, reducing their likelihood of relapse and promoting their recovery.

\section{References}

Allness, D. J., \& Knoedler, W.H. (1998) The PACT Model of Community-based Treatment for Persons with Severe and Persistent Mental Illnesses: A Manual for PACT Start-up. Arlington, VA: Programs of Assertive Community Treatment, Inc. (NAMI).

Bindman, J., Beck, A., Thornicroft, G., et al (2000) Psychiatric patients at greatest risk and in greatest need: impact of the Supervision Register Policy. British Journal of Psychiatry, $177,33-37$.

Department of Health (1990) Caring for People. The Care Programme Approach for People with a Mental Illness Referred to Specialist Mental Health Services. Joint Health/Social Services Circular. C(90)23/LASSL(90)11. London: Department of Health.

Department of Health (1995) Building Bridges: A Guide to Arrangements For Inter-agency Working for the Care and Protection of Severely Mentally Ill People. London: Department of Health.

Department of Health (1999) Effective Care Co-ordination in Mental Health Services: Modernizing the Care Programme Approach - A Policy Booklet. London: Department of Health.

Department of Health (2000) The NHS Plan (Cmnd 4818-I). London: Department of Health.

Department of Health (2002) Community Mental Health Teams. Mental Health Policy Implementation Guide. London: Department of Health.

Department of Health (2004) Guidance on New Ways of Working for Psychiatrists in a Multi-disciplinary and 
Multi-agency Context: National Steering Group. Interim Report. London: Department of Health.

Department of Health and Social Security (1988) Report of the Committee of Inquiry into the Care and After Care of Sharon Campbell (Chairman: J. Spokes). London: HMSO.

Franklin, D., Pinfold, V., Bindman, J., et al (2000) Consultant psychiatrists' experiences of using supervised discharge. Results of a national survey. Psychiatric Bulletin, 24, 412415

Health Services Research/Community Psychiatry (PRiSM) (2005) Threshold Assessment Grid (TAG). London: Institute of Psychiatry. http://www.iop.kcl.ac.uk/iopweb/ virtual $/$ ?path $=/ \mathrm{hsr} / \mathrm{prism} / \mathrm{tag} /$ -

Henderson, C., Flood, C., Leese, M., et al (2004) Effect of joint crisis plans on use of compulsory treatment in psychiatry: single blind randomised controlled trial. $B M J$ 329, 136 .

Kingdon, D. (1994a) Making care programming work. Advances in Psychiatric Treatment, 1, 41-46.

Kingdon D (1994b) Care programme approach, recent government policy and legislation. Psychiatric Bulletin, 18, $68-70$

Kingdon, D. (1998) Reclaiming the care programme approach. Psychiatric Bulletin, 22,

Royal College of Psychiatrists (2004) Good Psychiatric Practice (2nd edn) (Council Report CR125). London: Royal College of Psychiatrists.

Simpson, A., Miller, C. \& Bowers, L. (2003) The history of the Care Programme Approach in England: where did it go wrong? Journal of Mental Health, 12, 489-504.

Social Services Inspectorate (1999) Still Building Bridges. London: Department of Health.

\section{MCQs}

\section{The CPA is divided into:}

a unidisciplinary, bidisciplinary and multidisciplinary

b standard and enhanced

c levels 1, 2 and 3

d basic and step-up

e simple and complex.

2 A single point of entry has been advocated for the following reasons:

a limited financial resources and personnel

$\mathrm{b}$ to allow the psychiatrist to take on more of a consultant role

c to make the best use of 'medical' time

d to improve patient adherence to treatment plans

e to enhance carer involvement.
3 Supervision registers and supervised discharges have had an impact on CPA by:

a increasing the assessment of risk and needs of patients on the register

$b$ being used in the absence of CPA

c possibly having accelerated the process by which patients have been identified as requiring CPA

d making it more complex

e necessitating weekly reviews of the CPA.

4 With an increasing number of teams such as crisis resolution, early intervention and assertive outreach:

a there is less chance of patients falling through the net

$\mathrm{b}$ the team at the point of entry should initiate the CPA

c community mental health teams should take on more cases because they have low case-loads

d there should be a locally agreed framework for CPA

e it is necessary to ensure that patients meet the criteria for severe mental illness.

5 Local authorities that have made the most progress in implementing the guidance in Building Bridges had:

a pooled budgets

b carer-focused groups

c a strategic lead, with all involved professionals

d user-focused groups

e shared management protocols.

\section{MCQ answers}

\begin{tabular}{|c|c|c|c|}
\hline 1 & 2 & 3 & 4 \\
\hline$F$ & a $\mathrm{T}$ & a $\mathrm{T}$ & a $F$ \\
\hline $\mathrm{T}$ & b $\mathrm{T}$ & $b F$ & b $\mathrm{T}$ \\
\hline $\mathrm{F}$ & c F & c $\mathrm{T}$ & c F \\
\hline $\mathrm{F}$ & d F & d F & $\mathrm{d} T$ \\
\hline $\mathrm{F}$ & e $F$ & e $F$ & e $F$ \\
\hline
\end{tabular}

